\title{
The Troubling Link Between Non-alcoholic Fatty Liver Disease (NAFLD) and Extrahepatic Cancers (EHC)
}

\author{
Ajit Venniyoor ${ }^{1}$, Abdul Aziz Al Farsi ${ }^{2}$, Bassim Al Bahrani ${ }^{2}$ \\ 1. Medical Oncology, National Oncology Center, The Royal Hospital, Muscat, OMN 2. Oncology, National Oncology \\ Center, The Royal Hospital, Muscat, OMN
}

Corresponding author: Ajit Venniyoor, avenniyoor@gmail.com

\begin{abstract}
Non-alcoholic fatty liver disease (NAFLD) is a fast-spreading epidemic across the globe and has serious implications far beyond that of a "benign" liver condition. It is usually an outcome of ectopic fat storage due to chronic positive energy balance leading to obesity and is associated with multiple health problems. While association with cardiovascular disease and hepatocellular cancer is well recognized, it is becoming clear the NAFLD carries with it an increased risk of cancers of extrahepatic tissues. Studies have reported a higher risk for cancers of the colon, breast, prostate, lung, and pancreas. Fatty liver is associated with increased mortality; there is an urgent need to understand that fatty liver is not always benign, and not always associated with obesity. It is, however, a reversible condition and early recognition and intervention can alter its natural history and associated complications.
\end{abstract}

Review began $07 / 30 / 2021$ Review ended 08/16/2021 Published 08/20/2021

\section{(c) Copyright 2021}

Venniyoor et al. This is an open access article distributed under the terms of the Creative Commons Attribution License CC-BY 4.0., which permits unrestricted use, distribution, and reproduction in any medium, provided the original author and source are credited.
Categories: Endocrinology/Diabetes/Metabolism, Gastroenterology, Oncology

Keywords: extrahepatic cancers, diabetes type 2, adipose tissue, nafld, fatty liver, cancer, insulin resistance, ectopic fat storage, obesity-related cancers

\section{Introduction And Background}

Non-alcoholic fatty liver disease (NAFLD) is one of the commonest indicators of ill-health in the modern era of obesogenic lifestyle, next to obesity itself [1,2]. It affects a quarter of the world's population, with more than a billion individuals suffering from this condition worldwide [3,4]. NAFLD encompasses a wide spectrum of conditions ranging from "benign" fatty liver (hepatic steatosis, non-alcoholic fatty liver (NAFL), which is the accumulation of fat in more than $5 \%$ of hepatocytes), to non-alcoholic steatohepatitis (NASH, the inflammatory stage), to fibrosis, cirrhosis (with later decompensation), and hepatocellular carcinoma (HCC). The advanced stages are now the commonest cause of liver transplantation in the western world [5].

There are multiple causes for fatty liver, but the term NAFLD is conventionally restricted to that related to nutritional causes, obesity, and metabolic syndrome. Causes of fatty liver can be genetic (PNPLA3 mutation, lipodystrophies) [6], drugs [7], surgery (pancreatico-duodenectomy) [8], and hormonal (such as deficiency of thyroxine, estrogen, or testosterone) [9], but these are excluded while discussing NAFLD. There is a proposal to change the name from NAFLD to the more specific metabolic-associated fatty liver disease (MAFLD), which is scientifically accurate and removes the stigma associated with alcohol [10]. It must be noted that although NAFLD is thought to be a disease of the obese (reaching up to $50 \%$ in this population [11]), it is not necessary for a person to be overweight to have NAFLD ("lean NAFLD" [12]). However, it is clear that the vast majority of NAFLD is associated with metabolic and lifestyle disorders [13]).

\section{Review}

\section{Epidemiology}

The prevalence of NAFLD is disturbingly high across the world, varying between $25 \%$ and $35 \%$ globally. The highest prevalence is reported from South America (31\%) and Middle East (32\%), followed by Asia (27\%) and the USA (24\%), while the prevalence is lowest in Africa (14\%) [14]. The Middle East thus carries a heavy burden of NAFLD cases.

Recent figures suggest that more than $50 \%$ of the Omani population is overweight, and $30 \%$ have a body mass index (BMI) of more than 30\% [15]. Neighboring countries such as the United Arab Emirates (UAE) and Saudi Arabia which are also struggling with a high burden of obesity have reported a NAFLD prevalence of $25 \%$ [16,17], posing a significant economic burden [18]. A recent analysis of Global Burden of Disease data noted the increasing prevalence of NAFLD across all regions of the world from 1990 to 2017 and pointed out Oman as the country with the maximum increase [19]. Similarly, NASH as a cause of cirrhosis in Oman increased from an age-standardized rate (ASR) of 3.54 in 1990 to an ASR of 9.59 in 2017, a 540\% surge [20]. Among the 195 countries studied in this report, the highest growth rate of incident cases was found in the Gulf Cooperation Council countries with UAE leading with a whopping 1,119\% increase, followed by Qatar 
Surprisingly, in a study of HCC diagnosed from Oman between 2008 and 2015, of 284 patients, only two of 227 patients with cirrhosis $(0.9 \%)$ were reported to have a cryptogenic etiology [21] (potentially due to NASH), while alcoholic cirrhosis was much higher at 9.5\%. This highlights a vexing problem; overlapping conditions such as viral hepatitis in the case of NASH will lead to underestimation of NASH as a cause of cirrhosis and under-recognition of NASH as a cause of HCC.

\section{Mechanisms of NAFLD}

Steatosis occurs in hepatocytes due to two main mechanisms [22] - increased accumulation which is due to the overflow of free fatty acids (FFAs) from adipocyte tissue (which contributes to $60 \%$ of the stored fat), increased de novo lipogenesis (25\%) and FFA from the diet (15\%-20\%) and reduced output which is due to reduced FFA oxidation and reduced secretion of lipids. De novo lipogenesis is particularly affected by the consumption of fructose in "sugary" drinks [23].

The relation between obesity, hyperinsulinemia, insulin resistance, type 2 diabetes mellitus (T2DM), and NAFLD is a complex, "chicken and egg" situation. While the events that spark the inflammation that converts steatosis to steatohepatitis have been comprehensively reviewed [24], what causes the initial fat accumulation (simple steatosis) and insulin resistance is a matter of controversy. Few authors believe that insulin resistance causes NAFLD [25,26] while many suggest that NAFLD leads to insulin resistance and hyperinsulinemia $[27,28]$. The association is clear; a recent meta-analysis showed that NAFLD is associated with a 2.2 increased risk of T2DM [29]; the question is which comes first. This discussion is intimately tied to the controversy over the origins of obesity. The older "calorie in, calorie out" (CICO) or energy balance model is being challenged by the carbohydrate-insulin model (CIM) of obesity [30]. Current evidence suggests that constant positive energy balance in the modern obesogenic environment leads initially to hyperinsulinemia [31] in an effort to maintain normoglycemia. Indeed, it is known that insulin levels rise almost a decade before diabetes is detected [32-35] and are sometimes referred to as Stage 1 of diabetes associated with compensation [36].

Insulin is an anabolic, fat-storage hormone, and hyperinsulinemia results in varying degrees of obesity depending on the fat storage capacity of the individual (the "personal fat threshold" [37]). The latter is determined genetically or in utero. Fat is initially stored in the "safe" white adipose tissue (WAT), which is predominantly subcutaneous. Chronic positive energy intake leads to the excess fat (in form of FFAs) spilling over; the extra fat is stored in "unsafe" sites such as visceral adipose tissue (VAT) and liver (NAFLD) (ectopic fat storage). The outcome is insulin resistance (by lipid metabolites like diacylglycerol and ceramides), and T2DM [31]. NAFLD is further aggravated by fructose-containing “sugary” foods [23,38].

It is clear now that hyperinsulinemia is one of the earliest manifestations of modern-day chronic calorie excess/adiposity and appears much before the onset of insulin resistance and T2DM [39]. The modern highcarbohydrate diet with a high glycemic index and glycemic load is directly responsible for the raised insulin levels. Several studies have shown that low carbohydrate intake (which results in a ketogenic or "insulin sparing” diet) can reverse these changes and indeed, T2DM itself [40]. It is probable that fatty foods have been wrongly blamed for the obesity epidemic, and the sugar [41] and fast-food industry marketing ultraprocessed food [42] have actively contributed to this myth [43].

The hyperinsulinemic syndrome [44] has other manifestations, including cardiovascular, respiratory, and renal disorders, cancer, and reproductive diseases such as polycystic ovarian syndrome (PCOS); these can occur independently of obesity or T2DM. It is not clear whether these diseases are due to high levels of insulin per se, or other proximate causes.

\section{NAFLD and extrahepatic cancers (EHC)}

It was conventionally thought that the earliest manifestation of NAFLD, the fatty liver (NAFL), is a benign condition and becomes a medical problem only when it progresses to NASH, and later to fibrosis, cirrhosis with decompensation, and/or HCC. However, recent evidence suggests that the finding of hepatic steatosis is not benign and has significant health consequences [45]. NAFLD is an early warning indicator of extrahepatic disorders involving the cardiovascular [46], respiratory [47], renal [48], and reproductive system [49]. NAFLD is also associated with an increased risk of premalignant conditions such as colorectal polyps [50] but apparently not with ductal carcinoma in situ (DCIS). Several recent publications have now highlighted the risk of EHCs, something that has been underestimated so far [51].

In a prospective study from Rochester County, Minnesota, Allen et al. [52] followed 4,722 patients of NAFLD for a median of eight years and reported a 90\% higher risk of malignancy (2,224 incident cancers; RR 1.9), especially of liver, endometrium, stomach, pancreas, and colon. Surprisingly, obesity alone did not increase the relative risk (consistent with the idea that people with adequate safe storage space for fat may become obese but will remain healthy, the metabolically healthy obese or MHO). The authors suggest that as the association between obesity and cancer risk is small; NAFLD may be a mediator of the obesity-cancer association. 
In a long-term population-based cohort study from Sweden of 8,892 patients of biopsy-proven NAFLD [53], EHC was reported as a major cause of mortality. There is a higher risk of HCC across the spectrum of simple steatosis to cirrhosis as expected, and also a modest rise in EHCs (that of the pancreas, kidney/bladder, and melanoma)

A meta-analysis by Mantovani et al. [54] of 10 cohort studies with 8,485 incident cases of EHCs occurring over a median follow-up of 5.8 years reported that NAFLD was associated with a 1.5 -fold to twofold risk of GI cancers (esophagus, stomach, pancreas, and colorectal cancers), and 1.2-fold to 1.5-fold risk of lung, breast, gynecological and urinary system cancers. (Although lung cancer is not what one would expect in relation to obesity, a Chinese study [55] did report that both obesity and NAFLD are independent risk factors for adenocarcinoma of the lung, as did a Danish study [56].)

Another meta-analysis of 26 studies [57] has confirmed the association of NAFLD with EHCs, especially of colon, breast, esophagus, stomach, pancreas, prostate, and also cholangiocarcinomas.

A decade-long study from China [58] also showed that a significant cause of mortality in NAFLD is due to EHCs, second only to cardiovascular causes. In this study of more than 10,000 deaths during hospitalization, cardio- and cerebral vascular disease (CVD) ranked first in causes of death (35.6\%), followed by extrahepatic malignancies (22.6\%), infection (11.0\%), kidney disease (7.5\%), liver-related diseases (5.2\%), respiratory diseases (3.9\%), digestive diseases (3.5\%), endocrine diseases (3.5\%), and other diseases (7.2\%).

What is interesting is that obesity is not an essential requirement for EHCs associated with NAFLD. Obesityrelated cancers (ORCs) are a recognized entity [59], and the International Agency for Research on Cancer report in the New England Journal of Medicine listed 13 cancers with a higher incidence in obese individuals, especially that of the GIT and the reproductive system [60]. It is well known that BMI, which IARC used as its criteria, is an inadequate measure of unhealthy obesity, as it neither indicates the percentage of body fat mass nor the location of the fat [61], which determines the metabolic impact. Individuals with normal BMI but with "metabolic obesity" (sometimes called TOFI; thin outside, fat inside, an allusion to visceral obesity) have a higher cancer risk [62]. People at the opposite end of the spectrum, i.e., obese but apparently healthy (the MHO) are now noted to have excess mortality mainly due to cardiovascular causes; an excess risk of cancers has not been reported [63].

In Allen's study [52], NAFLD was associated with a higher risk of incident cancers (overall malignancy IRR=2.0), while obesity alone was not (IRR=1.0). Ectopic fat deposition, which cannot be measured by BMI, seems to be the common underlying factor in the etiopathogenesis of metabolic disorders and metabolic cancers.

Association is, of course, not causation and the larger question is, does fatty liver directly cause cancer? Causality is difficult to establish, and there could be another proximate cause for both fatty liver and cancer. Reversal of NAFLD leads to a drop in the incidence of cancers, the most effective way being bariatric surgery [64]. It is known that weight loss of 5\% reverses NAFLD. However, the Women Health Initiative (WHI) study indicates that while an intentional $5 \%$ weight loss reduced overall cancer risk (HR 0.88), and specifically that of endometrial cancer (HR 0.72), it did not reduce the risk of other ORCs such as of colon, breast, pancreas, kidney, thyroid, or liver [65]. The ultimate proof will probably come from long-term follow-up of patients specifically treated for NAFLD without other metabolic perturbations; this should indicate whether the ultimate cause lies in the liver or in the adipose tissue.

\section{Mechanisms of carcinogenesis}

The molecular links between obesity and cancer have been speculated on [66]; recent reviews have addressed the molecular mechanisms tying NAFLD to HCC [67] but the remote effects of NAFLD leading to EHC are less clear. The hormones, insulin and insulin-like growth factor-1 (IGF-1) have been implicated with some evidence [68].

The liver itself secretes a vast repertoire of hepatocytes [69,70], some of them with proliferative potentials such as FGF21 and fetuin-A [71]. To add to the complexity, several cytokines such as irisin [72] (mainly from skeletal muscles, another site of ectopic fat deposition), and leptin and adiponectin [73] (from adipose tissue) can alter tumor growth.

The mechanism(s) is much more than of academic interest as blocking the proliferative/mutagenic signals should (theoretically) uncouple NAFLD from its remote effects such as EHCs. This should be an area of research priority as it is unlikely that efforts to curb the incidence of NAFLD will bear fruit in near future.

\section{Implications}

Effect on Natural History

It is an intriguing question whether the presence of NAFLD alters the natural history of cancer. Some studies 
suggest that fatty liver increases the risk of liver metastasis (in lung cancer) [74], but others indicate no such effect (rectal cancer) [75] or even a reduced risk (in breast and colon cancer) [76,77].

Effect on Drug Response

Another evolving issue is the altered dynamics of immune checkpoint inhibitors (ICIs), in patients with NAFLD. ICIs themselves have been reported to cause NAFLD [78] and also cause drug-induced liver injury (DILI) in patients of NAFLD [79]; obese patients treated with ICIs also have a higher risk of thyrotoxicosis [80]. The better survival of some cancer patients (renal cell, melanoma, lung) if associated with obesity has been termed an "obesity paradox" [81]; this effect is consistent with a better response to ICIs in obese renal cell cancers patients [82]. However, a recent meta-analysis concluded that the current evidence does not support a clear positive association of BMI with survival outcomes [83]. Response to ICIs may be cancerspecific and even metastatic-site specific; one study concluded that HCC arising in a background of NASH is less responsive to ICIs [84], but liver metastasis of lung cancer responded better in NASH-affected livers [85]. Interestingly, liver metastasis from colorectal cancer has a higher risk of recurrence if resected from NASH-affected livers [86]. Further studies are needed but, in the meantime, Oncologists should be cautious while using this class of drugs in patients with NAFLD in view of a higher reported risk of DILI.

\section{High-Risk Warning}

At the general practitioner level, there should be increasing awareness that hepatic steatosis is not a benign condition; it should not be dismissed as an incidental finding in the ultrasound scan of the abdomen, as is done for renal or liver cysts. Firstly, about $20 \%$ of NAFL progress to NASH, and $40 \%$ of the latter go on to develop fibrosis; the risk of progression to NASH and fibrosis can be rapid, with NAFL progressing to fibrosis in an average of 14 years while NASH can in half that duration [2]; this can be dramatic in some. Secondly, identification of NAFLD on ultrasound (or by the asymptomatic rise in the liver enzyme ALT) demands to work up for associated conditions such as hypertension, T2DM, dyslipidemia, and other components of metabolic syndrome [2]. The higher risks of not just HCC but also of EHCs should be informed to the patient. The workup of such patients has been standardized and liver biopsy may be indicated in selected patients [1]. There should be an all-out effort to reverse it, starting with lifestyle changes, the simplest being to avoid sugary drinks. "Lean NAFLD" is not common and obesity remains overwhelmingly the largest cause of NAFLD. Despite social trends to avoid fat-shaming and accept obesity as the "new normal" [87], as doctors we must continue to campaign vigorously against obesity, as we did against smoking. This should include supporting legislation to make sugary and ultra-processed foods expensive and less accessible while subsidizing healthier options.

Recommendations for Screening?

It has been suggested that patients with NAFLD should be candidates for targeted screening for EHCs [52]. Three of the commonly associated cancers, that of colon, prostate, the breast are already in screening guidelines of many countries, and there should be an effort to include more in patients of NAFLD, such as endometrium. Prospective studies are required in this setting - especially to study the cost-effectiveness of screening and whether this leads to reduction of mortality - the only meaningful endpoint of screening studies.

\section{Management}

Reversal by Weight Loss

Reversal of NAFLD can either be by weight loss or by specifically targeting NAFLD. Weight loss through lifestyle modification (diet and exercise) is the most cost-effective intervention to reverse NAFLD [88,89] but decades of effort and billions of dollars later, it is clear that weight loss by dieting is easier said than done. Moderate weight reduction is highly effective at decreasing hepatic fat content [90], but 70\% to $95 \%$ of those who lose significant weight subsequently regain it [91]. The Endocrine Society's statement [92] on the pathogenesis of obesity noted that the recovery of lost weight is the largest single obstacle to effective longterm weight loss and identifying biological mechanisms that defend excess fat is a priority. Bariatric surgery remains the single most effective intervention to reverse obesity and its metabolic consequences including NAFLD and is associated with reduced risk of cancers including cancers of colon, pancreas, endometrium, thyroid, and of course, liver [93]; however, this procedure cannot be recommended for all as it comes with known morbidity and mortality, is cleared only for the morbidly obese and fails in about $15 \%$ of patients. The effects of bariatric surgery can be mimicked by the very low-calorie diet (VLCD) as shown by the DIRECT trial [94]. Adapting to a Mediterranean diet is a viable option to prevent and treat NAFLD [95]. GLP-1 receptor agonists such as liraglutide [96] and semaglutide [97] can produce up to $10 \%-15 \%$ weight loss and are increasingly used to manage NAFLD [98].

Targeted Reversal

Specific treatment of NAFLD is in its nascent stages [99]. It is clear that weight loss is not necessary to 
reverse NAFLD; NAFLD can be addressed with a low carbohydrate, high protein, high fiber diet [100,101], or fructose-limited diet [102]. Despite active research [103,104], there are very few pharmacological interventions in current guidelines [105]. Pioglitazone, vitamin E, and ursodeoxycholic acid (UDCA) are possible agents but their safety is a matter of concern [106]; more are in the pipeline [107].

An epidemiological study from China [108] gives indirect evidence that personal capacity of fat storage is related to the risk of NAFLD. Individual fat storage capacity is determined by the time of reaching adulthood and this study showed that exposure to famine conditions during the fetal or childhood stage (with minimal or no development of fat storage capacity) leads to a higher risk of NAFLD in adulthood. The implication is that promoting healthy energy storage in the face of caloric excess may lead to an uncoupling of obesity from NAFLD and the metabolic syndrome [109]. Expansion of subcutaneous "safe" WAT is a method of diverting fat away from the liver; though causing weight gain, this technique normalizes metabolic parameters and reverses NAFLD associated with obesity. This has been demonstrated experimentally in adiponectin overexpressing transgenic mice [110]. Pioglitazone [111,112] and other PPARY agonists act similarly and are effective in NAFLD; however, as can be anticipated, they cause weight gain.

Several agents that directly reverse NAFLD such as controlled-release mitochondrial protonophore (CRMP) are being studied [113]. Two rather unusual approaches are fecal microbiota transplantation [114] and whole-body vibration [115].

Finally, research is essential to discover the mechanisms linking NAFLD to EHCs so as to enable intervention. It is unlikely that we will achieve any form of control over the burgeoning obesity and NAFLD rates in near future [116]. The prevalence of NAFLD will continue to rise [117]. NAFLD is fueling an upsurge in cardiovascular diseases (CVD) [118] including hypertension [119]. Although a similar rise in T2DM has the potential to increase CVD, a recent study from the UK showed that death rates due to vascular diseases are actually declining in patients of T2DM (leaving cancer as now the leading cause of mortality) [120]. This was achieved not just by better glycemic control but by controlling other risk factors for vascular diseases, such as hypertension and dyslipidemia. Similarly, intense research is needed to identify additional risk factor(s) linking NAFLD to EHC, which can then be addressed.

\section{Conclusions}

Chronic positive energy balance overwhelms safe fat storage depots in subcutaneous WAT, leading to ectopic fat storage in unsafe sites such as VAT and liver. This causes insulin resistance, metabolic syndromes, and, as is increasingly clear, EHC. Clinicians and their patients should be made aware that fatty liver is just the tip of an iceberg; NAFLD is an indicator of an underlying plethora of metabolic disturbances. Aggressive measures must be instituted early to reverse it. Obesity and NAFLD will continue to rise and will overwhelm the health care system in a not-too-distant future. Current advice on the control of obesity such as lifestyle changes is a clear failure. It would be prudent to investigate the causative link(s) between NAFLD and EHCs with the ultimate aim of discovering methods to uncouple them.

\section{Additional Information}

\section{Disclosures}

Conflicts of interest: In compliance with the ICMJE uniform disclosure form, all authors declare the following: Payment/services info: All authors have declared that no financial support was received from any organization for the submitted work. Financial relationships: All authors have declared that they have no financial relationships at present or within the previous three years with any organizations that might have an interest in the submitted work. Other relationships: All authors have declared that there are no other relationships or activities that could appear to have influenced the submitted work.

\section{References}

1. Stefan N, Häring H-U, Cusi K: Non-alcoholic fatty liver disease: causes, diagnosis, cardiometabolic consequences, and treatment strategies. Lancet Diabetes Endocrinol. 2019, 7:313-24. 10.1016/s22138587(18)30154-2

2. Sheka AC, Adeyi O, Thompson J, Hameed B, Crawford PA, Ikramuddin S: Nonalcoholic steatohepatitis: a review. JAMA. 2020, 323:1175-83. 10.1001/jama.2020.2298

3. Mitra S, De A, Chowdhury A: Epidemiology of non-alcoholic and alcoholic fatty liver diseases. Transl Gastroenterol Hepatol. 2020, 5:16. 10.21037/tgh.2019.09.08

4. Younossi Z, Anstee QM, Marietti M, et al.: Global burden of NAFLD and NASH: trends, predictions, risk factors and prevention. Nat Rev Gastroenterol Hepatol. 2018, 15:11-20. 10.1038/nrgastro.2017.109

5. Bzowej NH: Nonalcoholic steatohepatitis: the new frontier for liver transplantation . Curr Opin Organ Transplant. 2018, 23:169-74. 10.1097/MOT.0000000000000502

6. Hooper AJ, Adams LA, Burnett JR: Genetic determinants of hepatic steatosis in man . J Lipid Res. 2011, 52:593-617. 10.1194/jlr.R008896

7. Jehl A, Cugnet-Anceau C, Vigouroux C, et al.: Acquired generalized lipodystrophy: a new cause of anti-PD-1 immune-related diabetes. Diabetes Care. 2019, 42:2008-10. 10.2337/dc18-2535

8. McGhee-Jez AE, Chervoneva I, Yi M, et al.: Nonalcoholic fatty liver disease after pancreaticoduodenectomy for a cancer diagnosis. J Pancreat Cancer. 2021, 7:23-30. 10.1089/pancan.2020.0006 
9. Gor R, Siddiqui NA, Wijeratne Fernando R, Sreekantan Nair A, Illango J, Malik M, Hamid P: Unraveling the role of hypothyroidism in non-alcoholic fatty liver disease pathogenesis: correlations, conflicts, and the current stand. Cureus. 2021, 13:e14858. 10.7759/cureus.14858

10. Eslam M, Newsome PN, Sarin SK, et al.: A new definition for metabolic dysfunction-associated fatty liver disease: an international expert consensus statement. J Hepatol. 2020, 73:202-9. 10.1016/j.jhep.2020.03.039

11. Liu J, Ayada I, Zhang X, et al.: Estimating global prevalence of metabolic dysfunction-associated fatty liver disease in overweight or obese adults [PREPRINT]. Clin Gastroenterol Hepatol. 2021, S1542-3565(21)002081. 10.1016/j.cgh.2021.02.030

12. Francque S, Wong VW: NAFLD in lean individuals: not a benign disease [PREPRINT] . Gut. 2021, 10.1136/gutjnl-2021-324162

13. Samuel VT, Shulman GI: Nonalcoholic fatty liver disease as a nexus of metabolic and hepatic diseases . Cell Metab. 2018, 27:22-41. 10.1016/j.cmet.2017.08.002

14. Araújo AR, Rosso N, Bedogni G, Tiribelli C, Bellentani S: Global epidemiology of non-alcoholic fatty liver disease/non-alcoholic steatohepatitis: what we need in the future. Liver Int. 2018, 38 Suppl 1:47-51. 10.1111/liv.13643

15. Health Ministry: Obesity in Oman: a major public health challenge. Ministry of Health, Government of Oman, Oman; 2021.

16. Alswat K, Sanai FM, Al-Hamoudi W, et al.: Clinical and metabolic characteristics of non-alcoholic fatty liver disease patients in Saudi Arabia: data from the Systematic Observatory Liver Disease (SOLID) Registry. Diabetes Metab Syndr Obes. 2021, 14:1167-75. 10.2147/DMSO.S300051

17. Alswat K, Aljumah AA, Sanai FM, et al.: Nonalcoholic fatty liver disease burden - Saudi Arabia and United Arab Emirates, 2017-2030. Saudi J Gastroenterol. 2018, 24:211-9. 10.4103/sjg.SJG_122_18

18. Sanai FM, Al Khathlan A, Al Fadhli A, et al.: Clinical and economic burden of nonalcoholic steatohepatitis in Saudi Arabia, United Arab Emirates and Kuwait [PREPRINT]. Hepatol Int. 2021, 10.1007/s12072-021-10182$\mathrm{X}$

19. Ge X, Zheng L, Wang M, Du Y, Jiang J: Prevalence trends in non-alcoholic fatty liver disease at the global, regional and national levels, 1990-2017: a population-based observational study. BMJ Open. 2020, 10:e036663. 10.1136/bmjopen-2019-036663

20. Zhai M, Liu Z, Long J, et al.: The incidence trends of liver cirrhosis caused by nonalcoholic steatohepatitis via the GBD study 2017. Sci Rep. 2021, 11:5195. 10.1038/s41598-021-84577-Z

21. Al-Naamani K, Al-Hashami Z, Al-Siyabi O, et al.: Hepatocellular carcinoma in Oman: an analysis of 284 cases. Sultan Qaboos Univ Med J. 2020, 20:e316-22. 10.18295/squmj.2020.20.03.011

22. Chakravarthy MV, Neuschwander-Tetri BA: The metabolic basis of nonalcoholic steatohepatitis. Endocrinol Diabetes Metab. 2020, 3:e00112. 10.1002/edm2.112

23. Roeb E, Weiskirchen R: Fructose and non-alcoholic steatohepatitis. Front Pharmacol. 2021, 12:634344. 10.3389/fphar.2021.634344

24. Arab JP, Arrese M, Trauner M: Recent insights into the pathogenesis of nonalcoholic fatty liver disease . Annu Rev Pathol. 2018, 13:321-50. 10.1146/annurev-pathol-020117-043617

25. Smith GI, Shankaran M, Yoshino M, et al.: Insulin resistance drives hepatic de novo lipogenesis in nonalcoholic fatty liver disease. J Clin Invest. 2020, 130:1453-60. 10.1172/JCI134165

26. Lonardo A, Bellentani S, Ratziu V, Loria P: Insulin resistance in nonalcoholic steatohepatitis: necessary but not sufficient - death of a dogma from analysis of therapeutic studies?. Expert Rev Gastroenterol Hepatol. 2011, 5:279-89. 10.1586/egh.11.19

27. Armandi A, Rosso C, Caviglia GP, Bugianesi E: Insulin resistance across the spectrum of nonalcoholic fatty liver disease. Metabolites. 2021, 11:155. 10.3390/metabo11030155

28. Shin SW, Lee SJ: Ectopic fat in insulin resistance, dyslipidemia, and cardiometabolic disease . N Engl J Med. 2014, 371:2237-8. 10.1056/NEJMc1412427

29. Mantovani A, Petracca G, Beatrice G, Tilg H, Byrne CD, Targher G: Non-alcoholic fatty liver disease and risk of incident diabetes mellitus: an updated meta-analysis of 501022 adult individuals. Gut. 2021, 70:962-9. 10.1136/gutjnl-2020-322572

30. Ludwig DS, Ebbeling CB: The carbohydrate-insulin model of obesity: beyond "Calories In, Calories Out" . JAMA Intern Med. 2018, 178:1098-103. 10.1001/jamainternmed.2018.2933

31. Roden M, Shulman GI: The integrative biology of type 2 diabetes . Nature. 2019, 576:51-60. 10.1038/s41586019-1797-8

32. Wiebe N, Ye F, Crumley ET, Bello A, Stenvinkel P, Tonelli M: Temporal associations among body mass index, fasting insulin, and systemic inflammation: a systematic review and meta-analysis. JAMA Netw Open. 2021, 4:e211263. 10.1001/jamanetworkopen.2021.1263

33. Tabák AG, Jokela M, Akbaraly TN, Brunner EJ, Kivimäki M, Witte DR: Trajectories of glycaemia, insulin sensitivity, and insulin secretion before diagnosis of type 2 diabetes: an analysis from the Whitehall II study. Lancet. 2009, 373:2215-21. 10.1016/S0140-6736(09)60619-X

34. Matthews DR, Levy JC: Impending type 2 diabetes. Lancet. 2009, 373:2178-9. 10.1016/s0140-6736(09)608655

35. Sagesaka H, Sato Y, Someya Y, et al.: Type 2 diabetes: when does it start? . J Endocr Soc. 2018, 2:476-84. 10.1210/js.2018-00071

36. Weir GC, Bonner-Weir S: Five stages of evolving beta-cell dysfunction during progression to diabetes . Diabetes. 2004, 53 Suppl 3:S16-21. 10.2337/diabetes.53.suppl_3.s16

37. Taylor R, Holman RR: Normal weight individuals who develop type 2 diabetes: the personal fat threshold . Clin Sci (Lond). 2015, 128:405-10. 10.1042/CS20140553

38. White PJ, Abdelmalek MF: Insights into metabolic mechanisms and their application in the treatment of NASH. Clin Liver Dis (Hoboken). 2021, 17:29-32. 10.1002/cld.1062

39. van Vliet S, Koh HE, Patterson BW, et al.: Obesity is associated with increased basal and postprandial $\beta$-cell insulin secretion even in the absence of insulin resistance. Diabetes. 2020, 69:2112-9. 10.2337/db20-0377

40. Merrill JD, Soliman D, Kumar N, Lim S, Shariff AI, Yancy WS Jr: Low-carbohydrate and very-lowcarbohydrate diets in patients with diabetes. Diabetes Spectr. 2020, 33:133-42. 10.2337/ds19-0070 
41. Alexander Bentley R, Ruck DJ, Fouts HN: U.S. obesity as delayed effect of excess sugar . Econ Hum Biol. 2020, 36:100818. 10.1016/j.ehb.2019.100818

42. Hall KD: Ultra-processed diets cause excess calorie intake and weight gain: a one-month inpatient randomized controlled trial of ad libitum food intake. Cell Metab. 2019, 30:67-77.e3. 10.31232/osf.io/w3zh2

43. Kearns CE, Schmidt LA, Glantz SA: Sugar industry and coronary heart disease research: a historical analysis of internal idustry documents. JAMA Intern Med. 2016, 176:1680-5. 10.1001/jamainternmed.2016.5394

44. Kelly CT, Mansoor J, Dohm GL, Chapman WH 3rd, Pender JR 4th, Pories WJ: Hyperinsulinemic syndrome: the metabolic syndrome is broader than you think. Surgery. 2014, 156:405-11. 10.1016/j.surg.2014.04.028

45. Tilg H, Targher G: NAFLD-related mortality: simple hepatic steatosis is not as 'benign' as thought . Gut. 2021, 70:1212-3. 10.1136/gutjnl-2020-323188

46. Kasper P, Martin A, Lang S, Kütting F, Goeser T, Demir M, Steffen HM: NAFLD and cardiovascular diseases: a clinical review. Clin Res Cardiol. 2021, 110:921-37. 10.1007/s00392-020-01709-7

47. Botello-Manilla AE, López-Sánchez GN, Chávez-Tapia NC, Uribe M, Nuño-Lámbarri N: Hepatic steatosis and respiratory diseases: a new panorama. Ann Hepatol. 2021, 24:100320. 10.1016/j.aohep.2021.100320

48. Byrne CD, Targher G: NAFLD as a driver of chronic kidney disease . J Hepatol. 2020, 72:785-801. 10.1016/j.jhep.2020.01.013

49. Singeap AM, Stanciu C, Huiban L, et al.: Association between nonalcoholic fatty liver disease and endocrinopathies: clinical implications. Can J Gastroenterol Hepatol. 2021, 2021:6678142. $10.1155 / 2021 / 6678142$

50. Bhatt BD, Lukose T, Siegel AB, Brown RS Jr, Verna EC: Increased risk of colorectal polyps in patients with non-alcoholic fatty liver disease undergoing liver transplant evaluation. J Gastrointest Oncol. 2015, 6:45968. 10.3978/j.issn.2078-6891.2015.050

51. Lonardo A, Roncucci L: The "obese liver" and gastrointestinal cancer risk. Transl Gastroenterol Hepatol. 2020, 5:44. 10.21037/tgh.2019.12.07

52. Allen AM, Hicks SB, Mara KC, Larson JJ, Therneau TM: The risk of incident extrahepatic cancers is higher in non-alcoholic fatty liver disease than obesity - A longitudinal cohort study. J Hepatol. 2019, 71:1229-36. 10.1016/j.jhep.2019.08.018

53. Simon TG, Roelstraete B, Sharma R, Khalili H, Hagström H, Ludvigsson JF: Cancer risk in patients with biopsy-confirmed nonalcoholic fatty liver disease: a population-based cohort study [PREPRINT]. Hepatology. 2021, 10.1002/hep.31845

54. Mantovani A, Petracca G, Beatrice G, Csermely A, Tilg H, Byrne CD, Targher G: Non-alcoholic fatty liver disease and increased risk of incident extrahepatic cancers: a meta-analysis of observational cohort studies. Gut. 2021, gutjnl-2021-324191. 10.1136/gutjnl-2021-324191

55. Zhu CY, Qu JC, Cao HX, Chen GY, Shi YH, Fan JG: Obesity and nonalcoholic fatty liver disease associated with adenocarcinoma in patients with lung cancer. Medicine (Baltimore). 2019, 98:e17098. 10.1097/MD.0000000000017098

56. Sørensen HT, Mellemkjaer L, Jepsen P, Thulstrup AM, Baron J, Olsen JH, Vilstrup H: Risk of cancer in patients hospitalized with fatty liver: a Danish cohort study. J Clin Gastroenterol. 2003, 36:356-9. 10.1097/00004836-200304000-00015

57. Liu SS, Ma XF, Zhao J, Du SX, Zhang J, Dong MZ, Xin YN: Association between nonalcoholic fatty liver disease and extrahepatic cancers: a systematic review and meta-analysis. Lipids Health Dis. 2020, 19:118. 10.1186/s12944-020-01288-6

58. Lin Y, Gong X, Li X, et al.: Distinct cause of death profiles of hospitalized non-alcoholic fatty liver disease: a 10 years' cross-sectional multicenter study in China. Front Med (Lausanne). 2020, 7:584396. 10.3389/fmed.2020.584396

59. Venniyoor A: Obesity-related cancers: the coming epidemic. Indian J Med Paediatr Oncol. 2020, 41:328-34. 10.4103/ijmpo.ijmpo_117_20

60. Lauby-Secretan B, Scoccianti C, Loomis D, Grosse Y, Bianchini F, Straif K: Body fatness and cancer-viewpoint of the IARC Working Group. N Engl J Med. 2016, 375:794-8. 10.1056/NEJMsr1606602

61. Ghesmaty Sangachin M, Cavuoto LA, Wang Y: Use of various obesity measurement and classification methods in occupational safety and health research: a systematic review of the literature. BMC Obes. 2018, 5:28. 10.1186/s40608-018-0205-5

62. Liu B, Giffney HE, Arthur RS, Rohan TE, Dannenberg AJ: Cancer risk in normal weight individuals with metabolic obesity: a narrative review. Cancer Prev Res (Phila). 2021, 14:509-20. 10.1158/1940-6207.CAPR20-0633

63. Zhou Z, Macpherson J, Gray SR, et al.: Are people with metabolically healthy obesity really healthy? A prospective cohort study of 381,363 UK Biobank participants [PREPRINT]. Diabetologia. 2021, 10.1007/s00125-021-05484-6

64. de Brito E Silva MB, Tustumi F, de Miranda Neto AA, Dantas AC, Santo MA, Cecconello I: Gastric bypass compared with sleeve gastrectomy for nonalcoholic fatty liver disease: a dystematic review and metaanalysis. Obes Surg. 2021, 31:2762-72. 10.1007/s11695-021-05412-y

65. Luo J, Hendryx M, Manson JE, et al.: Intentional weight loss and obesity-related cancer risk. JNCI Cancer Spectr. 2019, 3:pkz054. 10.1093/jncics/pkz054

66. Venniyoor A: The most important questions in cancer research and clinical oncology-Question 2-5. Obesity-related cancers: more questions than answers. Chin J Cancer. 2017, 36:18. 10.1186/s40880-0170185-8

67. Wegermann K, Hyun J, Diehl AM: Molecular mechanisms linking nonalcoholic steatohepatitis to cancer. Clin Liver Dis (Hoboken). 2021, 17:6-10. 10.1002/cld.1006

68. Hopkins BD, Goncalves MD, Cantley LC: Insulin-PI3K signalling: an evolutionarily insulated metabolic driver of cancer. Nat Rev Endocrinol. 2020, 16:276-83. 10.1038/s41574-020-0329-9

69. Meex RC, Watt MJ: Hepatokines: linking nonalcoholic fatty liver disease and insulin resistance. Nat Rev Endocrinol. 2017, 13:509-20. 10.1038/nrendo.2017.56

70. Watt MJ, Miotto PM, De Nardo W, Montgomery MK: The liver as an endocrine organ-linking NAFLD and insulin resistance. Endocr Rev. 2019, 40:1367-93. 10.1210/er.2019-00034 
71. Peter A, Kovarova M, Staiger H, et al.: The hepatokines fetuin-A and fetuin-B are upregulated in the state of hepatic steatosis and may differently impact on glucose homeostasis in humans. Am J Physiol Endocrinol Metab. 2018, 314:E266-73. 10.1152/ajpendo.00262.2017

72. Zhang D, Tan X, Tang N, Huang F, Chen Z, Shi G: Review of research on the role of irisin in tumors . Onco Targets Ther. 2020, 13:4423-30. 10.2147/OTT.S245178

73. Straub LG, Scherer PE: Metabolic messengers: adiponectin. Nat Metab. 2019, 1:334-9. 10.1038/s42255-0190041-z

74. Wu W, Liao H, Ye W, Li X, Zhang J, Bu J: Fatty liver is a risk factor for liver metastasis in Chinese patients with non-small cell lung cancer. PeerJ. 2019, 7:e6612. 10.7717/peerj.6612

75. Besutti G, Damato A, Venturelli F, et al.: Baseline liver steatosis has no impact on liver metastases and overall survival in rectal cancer patients. BMC Cancer. 2021, 21:253. 10.1186/s12885-021-07980-9

76. Wu W, Chen J, Ye W, Li X, Zhang J: Fatty liver decreases the risk of liver metastasis in patients with breast cancer: a two-center cohort study. Breast Cancer Res Treat. 2017, 166:289-97. 10.1007/s10549-017-4411-5

77. Murono K, Kitayama J, Tsuno NH, et al.: Hepatic steatosis is associated with lower incidence of liver metastasis from colorectal cancer. Int J Colorectal Dis. 2013, 28:1065-72. 10.1007/s00384-013-1656-2

78. Eigentler T, Lomberg D, Machann J, Stefan N: Lipodystrophic nonalcoholic fatty liver disease induced by immune checkpoint blockade. Ann Intern Med. 2020, 172:836-7. 10.7326/L19-0635

79. Sawada K, Hayashi H, Nakajima S, Hasebe T, Fujiya M, Okumura T: Non-alcoholic fatty liver disease is a potential risk factor for liver injury caused by immune checkpoint inhibitor. J Gastroenterol Hepatol. 2020, 35:1042-8. 10.1111/jgh.14889

80. Pollack R, Ashash A, Cahn A, Rottenberg Y, Stern H, Dresner-Pollak R: Immune checkpoint inhibitorinduced thyroid dysfunction is associated with higher body mass index. J Clin Endocrinol Metab. 2020, 105:dgaa458. 10.1210/clinem/dgaa458

81. Lennon H, Sperrin M, Badrick E, Renehan AG: The obesity paradox in cancer: a review . Curr Oncol Rep. 2016, 18:56. 10.1007/s11912-016-0539-4

82. Lalani AA, Bakouny Z, Farah S, Donskov F, Dudani S, Heng DY, Choueiri TK: Assessment of immune checkpoint inhibitors and genomic alterations by body mass index in advanced renal cell carcinoma. JAMA Oncol. 2021, 7:773-5. 10.1001/jamaoncol.2021.0019

83. Indini A, Rijavec E, Ghidini M, et al.: Impact of BMI on survival outcomes of immunotherapy in solid tumors: a systematic review. Int J Mol Sci. 2021, 22:2628. 10.3390/ijms22052628

84. Pfister D, Núñez NG, Pinyol R, et al.: NASH limits anti-tumour surveillance in immunotherapy-treated HCC. Nature. 2021, 592:450-6. 10.1038/s41586-021-03362-0

85. Zhou J, Zhou F, Chu X, et al.: Non-alcoholic fatty liver disease is associated with immune checkpoint inhibitor-based treatment response in patients with non-small cell lung cancer with liver metastases. Transl Lung Cancer Res. 2020, 9:316-24. 10.21037/tlcr.2020.04.15

86. Molla NW, Hassanain MM, Fadel Z, et al.: Effect of non-alcoholic liver disease on recurrence rate and liver regeneration after liver resection for colorectal liver metastases. Curr Oncol. 2017, 24:e233-43. 10.3747/co.24.3133

87. Flint SW: Time to end weight stigma in healthcare. EClinicalMedicine. 2021, 34:100810. 10.1016/j.eclinm.2021.100810

88. Vilar-Gomez E, Martinez-Perez Y, Calzadilla-Bertot L, et al.: Weight loss through lifestyle modification significantly reduces features of nonalcoholic steatohepatitis. Gastroenterology. 2015, 149:367-78.e5; quiz e14-5. 10.1053/j.gastro.2015.04.005

89. Takahashi H, Kotani K, Tanaka K, Egucih Y, Anzai K: Therapeutic approaches to nonalcoholic fatty liver disease: exercise itervention and related mechanisms. Front Endocrinol (Lausanne). 2018, 9:588. 10.3389/fendo.2018.00588

90. Hydes TJ, Ravi S, Loomba R, Gray ME: Evidence-based clinical advice for nutrition and dietary weight loss strategies for the management of NAFLD and NASH. Clin Mol Hepatol. 2020, 26:383-400. 10.3350/cmh.2020.0067

91. Hall KD, Kahan S: Maintenance of lost weight and long-term management of obesity. Med Clin North Am. 2018, 102:183-97. 10.1016/j.mcna.2017.08.012

92. Schwartz MW, Seeley RJ, Zeltser LM, Drewnowski A, Ravussin E, Redman LM, Leibel RL: Obesity pathogenesis: an endocrine society scientific statement. Endocr Rev. 2017, 38:267-96. 10.1210/er.201700111

93. Rustgi VK, Li Y, Gupta K, Minacapelli CD, Bhurwal A, Catalano C, Elsaid MI: Bariatric surgery reduces cancer risk in adults with nonalcoholic fatty liver disease and severe obesity. Gastroenterology. 2021, 161:17184.e10. 10.1053/j.gastro.2021.03.021

94. Lean MEJ, Leslie WS, Barnes AC, et al.: Durability of a primary care-led weight-management intervention for remission of type 2 diabetes: 2-year results of the DiRECT open-label, cluster-randomised trial. Lancet Diabetes Endocrinol. 2019, 7:34455. 10.1016/s2213-8587(19)30068-3

95. Gosal H, Kaur H, Chakwop Ngassa H, Elmenawi KA, Anil V, Mohammed L: The significance of the Mediterranean diet in the management of non-alcoholic fatty liver disease: a systematic review. Cureus. 2021, 13:e15618. 10.7759/cureus.15618

96. Alruwaili H, Dehestani B, le Roux CW: Clinical impact of liraglutide as a treatment of obesity . Clin Pharmacol. 2021, 13:53-60. 10.2147/CPAA.S276085

97. Newsome PN, Buchholtz K, Cusi K, et al.: A placebo-controlled trial of subcutaneous semaglutide in nonalcoholic steatohepatitis. N Engl J Med. 2021, 384:1113-24. 10.1056/NEJMoa2028395

98. Ghazanfar H, Kandhi SD, Nawaz I, et al.: Role of glucagon-like peptide-1 receptor agonists in the management of non-alcoholic steatohepatitis: a clinical review article. Cureus. 2021, 13:e15141. 10.7759/cureus. 15141

99. Ramanan SP, Mohamed MW, Aung SS, Sange I, Hamid P: Treatment of fatty liver disease: the present and the future. Cureus. 2021, 13:e12713. 10.7759/cureus.12713

100. Worm N: Beyond body weight-loss: dietary strategies targeting intrahepatic fat in NAFLD . Nutrients. 2020, 12:1316. 10.3390/nu12051316 
101. Risi R, Tozzi R, Watanabe M: Beyond weight loss in nonalcoholic fatty liver disease: the role of carbohydrate restriction. Curr Opin Clin Nutr Metab Care. 2021, 24:349-53.

10.1097/MCO.0000000000000762

102. Simons N, Veeraiah P, Simons PI, et al.: Effects of fructose restriction on liver steatosis (FRUITLESS); a double-blind randomized controlled trial. Am J Clin Nutr. 2021, 113:391-400. 10.1093/ajcn/nqaa332

103. Vuppalanchi R, Noureddin M, Alkhouri N, Sanyal AJ: Therapeutic pipeline in nonalcoholic steatohepatitis. Nat Rev Gastroenterol Hepatol. 2021, 18:373-92. 10.1038/s41575-020-00408-y

104. Goedeke L, Perry RJ, Shulman GI: Emerging pharmacological targets for the treatment of nonalcoholic fatty liver disease, insulin resistance, and type 2 diabetes. Annu Rev Pharmacol Toxicol. 2019, 59:65-87. 10.1146/annurev-pharmtox-010716-104727

105. Chalasani N, Younossi Z, Lavine JE, et al.: The diagnosis and management of nonalcoholic fatty liver disease: Practice guidance from the American Association for the Study of Liver Diseases. Hepatology. 2018, 67:328-57. 10.1002/hep.29367

106. Sanyal AJ, Chalasani N, Kowdley KV, et al.: Pioglitazone, vitamin E, or placebo for nonalcoholic steatohepatitis. N Engl J Med. 2010, 362:1675-85. 10.1056/NEJMoa0907929

107. Gawrieh S, Noureddin M, Loo N, et al.: Saroglitazar, a PPAR- $\alpha / \gamma$ agonist, for treatment of NAFLD: a randomized controlled double-blind phase 2 trial [PREPRINT]. Hepatology. 2021, 10.1002/hep.31843

108. Qi H, Hu C, Wang S, et al.: Early life famine exposure, adulthood obesity patterns and the risk of nonalcoholic fatty liver disease. Liver Int. 2020, 40:2694-705. 10.1111/liv.14572

109. Vishvanath L, Gupta RK: Contribution of adipogenesis to healthy adipose tissue expansion in obesity . J Clin Invest. 2019, 129:4022-31. 10.1172/JCI129191

110. Kim JY, van de Wall E, Laplante M, et al.: Obesity-associated improvements in metabolic profile through expansion of adipose tissue. J Clin Invest. 2007, 117:2621-37. 10.1172/JCI31021

111. de Souza CJ, Eckhardt M, Gagen K, Dong M, Chen W, Laurent D, Burkey BF: Effects of pioglitazone on adipose tissue remodeling within the setting of obesity and insulin resistance. Diabetes. 2001, 50:1863-71. 10.2337/diabetes.50.8.1863

112. Panunzi S, Maltese S, Verrastro O, et al.: Pioglitazone and bariatric surgery are the most effective treatments for non-alcoholic steatohepatitis: a hierarchical network meta-analysis. Diabetes Obes Metab. 2021, 23:980-90. 10.1111/dom.14304

113. Goedeke L, Shulman GI: Therapeutic potential of mitochondrial uncouplers for the treatment of metabolic associated fatty liver disease and NASH. Mol Metab. 2021, 46:101178. 10.1016/j.molmet.2021.101178

114. Delaune V, Orci LA, Lacotte S, Peloso A, Schrenzel J, Lazarevic V, Toso C: Fecal microbiota transplantation: a promising strategy in preventing the progression of non-alcoholic steatohepatitis and improving the anticancer immune response. Expert Opin Biol Ther. 2018, 18:1061-71. 10.1080/14712598.2018.1518424

115. Oh S, Oshida N, Someya N, et al.: Whole-body vibration for patients with nonalcoholic fatty liver disease: a 6-month prospective study. Physiol Rep. 2019, 7:e14062. 10.14814/phy2.14062

116. Gribble FM, O'Rahilly S: Obesity therapeutics: the end of the beginning. Cell Metab. 2021, 33:705-6. 10.1016/j.cmet.2021.03.012

117. Estes C, Razavi H, Loomba R, Younossi Z, Sanyal AJ: Modeling the epidemic of nonalcoholic fatty liver disease demonstrates an exponential increase in burden of disease. Hepatology. 2018, 67:123-33. 10.1002/hep.29466

118. Cai J, Zhang XJ, Ji YX, Zhang P, She ZG, Li H: Nonalcoholic fatty liver disease pandemic fuels the upsurge in cardiovascular diseases. Circ Res. 2020, 126:679-704. 10.1161/CIRCRESAHA.119.316337

119. Zhao YC, Zhao GJ, Chen Z, She ZG, Cai J, Li H: Nonalcoholic fatty liver disease: an emerging driver of hypertension. Hypertension. 2020, 75:275-84. 10.1161/HYPERTENSIONAHA.119.13419

120. Pearson-Stuttard J, Bennett J, Cheng YJ, Vamos EP, Cross AJ, Ezzati M, Gregg EW: Trends in predominant causes of death in individuals with and without diabetes in England from 2001 to 2018: an epidemiological analysis of linked primary care records. Lancet Diabetes Endocrinol. 2021, 9:165-73. 10.1016/S22138587(20)30431-9 